\title{
Considerations Regarding Embryo Culture Conditions: From Media to Epigenetics
}

\author{
MARA SIMOPOULOU ${ }^{1,2^{*}}$, KONSTANTINOS SFAKIANOUDIS $^{3 *}$, ANNA RAPANI $^{1}$, \\ POLINA GIANNELOU ${ }^{1,3}$, GEORGE ANIFANDIS ${ }^{4}$, STAMATIS BOLARIS ${ }^{5}$, \\ AGNI PANTOU $^{3}$, MARIA LAMBROPOULOU ${ }^{6}$, ATHANASIOS PAPPAS ${ }^{3}$, \\ EFTHIMIOS DELIGEOROGLOU ${ }^{2}$, KONSTANTINOS PANTOS $^{3 * *}$ and MICHAEL KOUTSILIERIS ${ }^{1 * *}$ \\ ${ }^{1}$ Department of Physiology, Medical School, National and Kapodistrian University of Athens, Athens, Greece; \\ ${ }^{2}$ Assisted Conception Unit, 2nd Department of Obstetrics and Gynecology, Aretaieion Hospital, \\ Medical School, National and Kapodistrian University of Athens, Athens, Greece; \\ ${ }^{3}$ Centre for Human Reproduction, Genesis Athens Clinic, Athens, Greece; \\ ${ }^{4}$ Department of Histology and Embryology, Faculty of Medicine, University of Thessaly, Larissa, Greece; \\ ${ }^{5}$ Assisted Conception Unit, Elena Venizelou General-Maternity District Hospital, Athens, Greece; \\ ${ }^{6}$ Department of Histology and Embryology, Medical School, \\ Democritus University of Thrace, Alexandroupolis, Greece
}

\begin{abstract}
There are numerous reports on embryo culture media and conditions in the laboratory, as the subject is multifaceted and complex, reflecting the variation in practice. In this scoping review, we attempt to approach the topic of culture media and conditions from the practitioners' perspective aiming to highlight, in a comprehensive fashion, important aspects regarding the options available, introduce points of debate and controversy, while maintaining the viewpoint of the practicing embryologist's concerns.
\end{abstract}

It has been suggested by numerous studies that the culture media employed during in vitro fertilization (IVF) cycles can influence implantation as well as pregnancy rates due to their effect on embryo quality (1-3). More evidence supporting this hypothesis has emerged. However, the connection

This article is freely accessible online.

*Joint first authorship; **joint last authorship.

Correspondence to: Mara Simopoulou, Assistant Professor of Physiology, Clinical Embryologist/Geneticist, Department of Physiology-Department of Obstetrics and Gynaecology, Medical School, National and Kapodistrian University of Athens 75, Mikras Asias 11527, Athens, Greece. E-mail: marasimopoulou@hotmail.com; info@pantos.gr

Key Words: Embryo culture media, conditions, trends, epigenetics, review. between medium composition and outcomes of IVF is still under investigation (4-8). A main reason why IVF results have improved in recent decades is considered to be the evolution of embryo culture systems.

There has been an enormous contribution from studies conducted on mammals in the past 50 years. Defining the optimal embryo culture medium historically started from an effort to mimic the composition of tubal fluid (9). The strides achieved since include considerations of the precise metabolic requirements as well as the medium's influence on gene expression, from the oocyte to the blastocyst. One of the main considerations is that media are being designed based on research of animal models, subsequently leading to question being asked about their true effect on human embryology (10). Commercially available media are tested based on mouse embryo performance $(11,12)$. In vivo, human embryos survive and adjust to a range of variable conditions provided by the mother during the gestational period. As the variation among commercial media designed for human embryo use is extensive, it is understandable why the 'perfect' culture media composition mirroring the in vivo exposure and support has not yet been manufactured.

\section{Key Considerations Regarding Culture Media}

Media were first produced in-house with limited quality control, now they are commercially and widely available containing nutrients, vitamins and growth factors and strictly produced by manufacturers who follow specific quality 
standards $(13,14)$. Although commercially available media have been widely used by embryologists, the production of in-house synthetic sequential media offers some unique advantages (15). Conducting an effective quality control is realistic, allowing the laboratory to abide by a strict protocol of choice. Adding or removing specific components is possible and finally, the IVF program is totally independent and capable of accommodating unexpected situations, such as a sudden influx of an increased number of IVF patients. However, the variation between different batches of in-house media is a major issue to be addressed $(13,16,17)$.

Despite the long-lasting debate regarding the use of commercial media versus in-house produced sequential media, in practice this dilemma has been resolved in favor of continuous commercially made single media. However, revisiting the synthetic in-house option, it seems that certain advantages could still be offered. A study performed by Yoon et al. showed that there was no significant difference between the in-house sequential media and the commercially available ones that were tested regarding implantation rates, pregnancy and the proportion of cycles with cryopreservation (15). However, the practical matters on producing in-house media still stand and therefore it represents as an unrealistic option for IVF laboratories of today to choose and invest in in-house media rather than the commercially available ones, with the security of testing and certification provided.

The question of sequential versus single-step medium culture has challenged embryologists in recent years. Nevertheless, without solid evidence wholly supporting either option, it is a choice every laboratory should make according to its needs. In a sequential system, one type of medium is used until day 3 of development and this medium is then replaced by one with a different composition until day 5-6. In contrast, the single culture system only requires the use of one type of medium throughout the entire culture process (18). The latter has been proposed as possibly being beneficial since it allows embryos to regulate their own microenvironment, while simultaneously it facilitates workload by removing an extra step in the culture process (19). The option of accommodating single medium culture by renewing it on day 3 should also be noted (20). There is a trend for going back to the original concept in improved versions of continuous culture media, represented by onestep media, a common practice within the IVF set-up. However, opposing arguments remain as the complete understanding of the metabolic profile of the human embryo and its needs is still an ongoing process and the answers defining synthesis of commercial media originate from nonhuman embryo culture.

Most of the media produced by manufactures are CE (European Conformity) and MEA (Mouse Embryo Assay) marked. CE marking is accompanied by full disclosure of the medium's precise composition, an assessment of its quality, post-market surveillance, systems for reporting and examining adverse incidents, and traceability (21). On the other hand, MEA marking is indicative of successful testing for $\geq 80 \%$ of mouse embryo development to blastocyst stage $(22,23)$ and has been widely criticized as it basically only reassures against toxicity issues rather than compatibility with human embryo development $(21,24-26)$. However, the actual number of embryos is not reported which may raise an issue regarding the validity of the results reported. For every batch of culture medium available, a certificate of analysis is provided, if requested, or can even be found online. Nevertheless, even when it comes to simple aspects of practices and protocols suggested for embryo culture, companies have agreed to disagree on what the optimal practice worth adopting is, as Vajta et al. have stated (19). This fact could potentially act as a compromising factor for IVF laboratory practice. For instance, the culture may not solely rely on media from a single company but rather employ media formulated by two or more different companies, which may be a usual practice especially regarding various stages throughout the culture. For example, employing media from a specific company for the procedures involved until the fertilization check, and from another company for the procedures involved until the culture stage, might be a practice that requires further investigation. There are still reports to be published on cycle results comparing media from one company for all procedures during the IVF cycle to media from different companies employed at various stages during culture. This results in great difficulty for any assessment regarding the potential impact for cases where combining media of different commercial origins for different steps of the IVF cycle is a standard practice. Besides the importance of the components listed in culture media, another aspect to take into consideration is the interactions among them, the impact of which on human embryo viability is unknown (27).

There is a limited number of companies providing culture media to the global IVF market. A detailed description of the components of those media is not always disclosed by the companies. Even though the main components of most commercial culture media are known, the definitive composition is complex including components such as growth factors, amino acids, serum supplements, salts, antibiotics, energy substrates, vitamins, buffer solutions, nucleotides, as well as trace elements and nuclease inhibitors, all at an undefined concentration $(28,29)$. One of the main issues is the fact that the concentrations are unknown and may vary considerably, highlighting the diversity of the culture environment and subsequently the varied conditions that the embryos are exposed to. Further investigations should focus on outcomes from different IVF laboratories employing different culture media. Such a defined approach would be welcomed by the scientific 
community of clinical embryologists. Meta-analyses on these data could assist towards highlighting differences. This is a possibility that could be worth exploring.

It has been reported that commercial culture media can affect the perinatal outcome, such as gestational age and birthweight (30). Animal studies have stated that specific components of culture media are linked to abnormal fetal growth $(31,32)$. On the other hand, there is contradicory evidence in regards to neonatal birth weight and culture media effects in humans. Dumoulin et al. reported that use of certain commercial culture media is significantly correlated with increased birth weight compared to other media (4), which has further been confirmed (5). However, others report no association between particular culture media and neonatal birthweight $(29,33-35)$. An overall statement is that the nutritional composition of the culture medium has an impact on early embryonic development. Based on principles of reproductive physiology, it has been proposed that maternal nutrition affects birth weight as well as metabolic and cardiovascular health $(36,37)$. The hypothesis that the composition of culture media is so crucial that it could possibly even affect offspring health may stem from that very fact.

Albeit a challenging practice, tighter regulation regarding the use of IVF culture media is required, especially when there is no legal binding for manufacturers to disclose an exact and all-inclusive report of a medium's composition. This should change in the future, as the lack of knowledge regarding the concentration of bioactive compounds may result in ineffective evaluation of potential risks.

Data regarding fertilization rate, embryo quality, implantation, pregnancy and live birth rates have to be closely evaluated and examined. A protocol for conducting follow-ups in regards to offspring health could be established at every IVF unit and be incorporated as an important quality-control marker. There is indisputably a risk for human embryos in the laboratory. Changing the composition of culture media should be performed after weighing and assessing risks and potential benefits. Another approach in theory to avoid risks related to culture media exposure could be shortening the period of in vitro culture. This could reduce the risk for the embryos at least in theory. However, this theoretical risk may be heightened in light of the growing practice of blastocyst culture, even though in practice, prolonged culture to the blastocyst stage is related to higher success rates (38). These favorable results associated with blastocyst culture are indisputable proof of the major developments in culture conditions, along with the concurrent improvement of commercially available culture medium formulations. In view of the variations in practice from protocols, to types of culture, to media availability from various companies enabling different approaches, a common universal approach promising optimal results is not realistic nor attainable for the practicing embryologist. Perhaps the consensus should focus on embracing the different options. To strengthen this approach, results and follow-up on the laboratory success rates extending to clinical data should be carefully monitored and analyzed for every practice. Extrapolating on the above, an overall statement could be that key performance indicators and success rates coupled with pediatric data should be recorded and examined to shape the optimal protocols for each practicing IVF laboratory.

\section{Principal Elements of Culture Media}

Concentrating on culture media translates into analyzing basic aspects such as medium components, the conditions they are exposed to, and the biochemistry regarding their optimal performance in the laboratory. The addition of select amino acids, glucose, phosphatase, insulin, trace elements, and antibiotics are some of the numerous factors aiming to serve as 'protectors' and 'promoters' enriching the potential of culture media (27). The following section refers to certain components that have managed to raise controversy and debate within the world of assisted reproductive technology (ART) with respect to the justification behind winning and securing their place in media formulations.

One of the basic components in culture media reportedly included to promote human embryo development are peptide growth factors. The matter of whether growth factors should be employed in routine IVF practice has already been raised (39). The possible detriments of enriching culture media with 'promising' elements should always be considered. In the attempt to fully examine the pathways that growth factors act on during embryo development, it has been revealed that the addition of specific growth factors affects gene expression at the blastocyst stage. Specifically, it was suggested that they affect gene expression while presenting unexpected effects when interacting with each other in the culture medium (40). One well-known effect that has translated into a disadvantage is the ability of growth factors to block apoptosis (41). Apoptosis serves a great role by eradicating genetically abnormal cells during embryo development, hence blocking this process by adding growth factors could be damaging. When additions to the basic medium formulation come along with a promising but controversial profile, every effort should be made to effectively weigh the pros and cons and make an informed decision. The appeal of better results alone is certainly not enough to act as the driver in making such decisions regarding medium formulation.

Another crucial medium element is the protein source. The most commonly used protein source in media for human embryo culture is albumin. It is a source of hormones, energy, vitamins, and various other factors, while it maintains $\mathrm{pH}$ buffer function, membrane stabilization, 
osmotic pressure and scavenging of toxic substances. Finally, it prevents embryos from adhering to the culture dish (42). Regarding humans, the effect of protein source/human serum albumin was first investigated by Zhu et al., who concluded that the protein source affected birthweight in humans (30). None of the previous studies had ever confirmed such a hypothesis, partially due to the selection of other culture media used in other research explaining those discrepancies. Furthermore, still light needs to be shed in order to determine which protein source is the safest for human culture without negatively affecting the embryo.

Addition of hyaluronic acid, on the other hand, is an intervention originating from the fact that depending on the day of the cycle, this component is present in the endometrium (43), and its receptors have also been detected in the preimplantation embryo $(44,45)$. This type of enriched culture medium has been suggested not to provide any major benefits on IVF outcome. According to Fancsovits et al., the addition of hyaluronic acid did not have any beneficial effect on the pregnancy nor the implantation or the delivery rates. However, they reported higher birth weight when hyaluronic acid was used at high concentration (42).

The question still remains with respect to the bioethics of employing factors in the set-up of embryo culture. These improving factors may on one hand present appealing improvements regarding development, but on the other hand they may cast a shadow with respect to the overall consequence on embryonic identity and its expression. It is, therefore, critical to select carefully and be aware of full media constitution so that the IVF laboratory can make an informed decision.

\section{The Consensus on Incubator Use}

Incubators are without a doubt one of the most important pieces of equipment in the laboratory, monitoring the environment and accommodating the embryos for their in vitro life. The present variety of incubators available featuring numerous characteristics and means of controlling their internal environment is due to technological advances initiated by the continuous enhancement of the IVF scientific community's understanding of what entails the optimal conditions. Understanding the variations of incubators and schools of thought on culture plays a pivotal role in enabling the IVF laboratory to proceed to an informed decision regarding the options available.

Incubators control the environmental variables which affect embryo development and act as potential stressors, namely temperature, $\mathrm{pH}$ of the culture medium, medium osmolality and air quality, which are regulated by gas concentrations, temperature and humidity (46). The Holy Grail of an incubator is to achieve stability, while the various mechanisms of achieving this present advantages and disadvantages. The welcoming of new technologies in an IVF laboratory is usually first taken for the incubators, thus choosing an incubator capable of adapting to constant changes is a must- have.

The IVF community has favored the bench-top incubator in practice in view of the comparative studies associating its use with improved embryo culture environment and potentially better results $(47,48)$. Bench-top incubators are the only type explicitly designed for clinical IVF use. The difficulties related to their use revolve around some limitations on quality control such as $\mathrm{pH}$ measurement of the stored culture media (46). However, conventional incubators still hold their place in the IVF laboratory, perhaps targeting media calibration and concentrating on sperm and oocyte preparation.

Determining the optimal selection of an incubator is still problematic, as there are no defined advantages enabling preference of one specific type over another. Adopting a mixture of different incubator types may be the wiser option for an up-to-date laboratory (46). New trends could be challenging for the practicing embryologists to embrace in the IVF set-up, however, it is crucial that benefits must be clearly documented in order for changes within the clinical practice to be realized. The matter of the learning curve needs to be taken into account, as well as time required for a new practice to be established effectively.

\section{Air Quality in Embryo Culture}

An additional factor worth considering in embryo culture is the $\mathrm{pH}$. The $\mathrm{pH}$ depends on the gases comprising the atmosphere in the culture incubator, which explains the reasoning behind securing an optimal gas atmosphere for embryo culture in order to protect gamete function and embryo development (49). Therefore, the oxygen concentration in the incubator should be taken into account in an IVF laboratory. Why oxygen concentration is so crucial in the way a culture works, besides its implication in the regulation of $\mathrm{pH}$, can be explained by investigations on the effect of oxidative stress on embryos and the increased production of reactive oxygen species (ROS) at certain gas concentrations $(50,51)$. ROS are by-products of aerobic respiration and metabolism that could potentially result in DNA and protein damage (52).

In vivo, embryos are exposed to oxygen concentrations that range from $2-8 \%$ (53). Culture conditions consisting of 3 . $5 \%$ oxygen have been shown to lead to higher rates of fertilization and cleavage (54). One major reason why a low oxygen tension is associated with improved embryo viability is possibly the effect it exerts on increasing expression of antioxidant enzymes and glucose transporters. Our understanding is predominantly limited regarding the mechanisms behind the benefits of culturing at a lower 
oxygen tension $(5 \%)$, but that it leads to higher success rates is a fact (55). In addition, it has been suggested that the oxygen concentration is a crucial clinical factor associated with global placental methylation and in turn epigenetics (56).

Other than hypoxic incubators, the overall quality of air circulating in the IVF laboratory is crucial. Air quality is an additional variable that affects in vitro embryo development, translating as the amount of volatile organic compounds (VOCs) present in the air (57-60). Thus, specialized air systems that can filter out VOCs from the laboratory atmosphere are utilized in order to achieve optimal air quality $(59,61)$. It is suggested and has been adopted in various practices that safe conditions regarding air quality should be secured within the incubator as well as outside via use of VOC-free inline gas filtration systems (46), or by employing a UV light source that for photocatalytic breakdown of VOCs in recirculating air (62). However, the efficiency of this practice is a matter of debate, while its application to benchtop incubators is problematic (46).

Do all of the above facts and statements really matter? They do and there is one study to rule them all. Heitmann et al. made a remarkable observation when they preserved important aspects of their IVF laboratory, including equipment, personnel and protocols, during relocation of their facility while improving only the environmental variables. Enhancing the quality of IVF laboratory conditions and air systems had profound positive effects on laboratory measures and patient outcomes regarding implantation and live birth (63).

The importance of air quality and adopting practices to entire optimal air quality through employing a hypoxic incubator and installing VOC filtration systems is irrefutably a step in the right direction. However, quality is more than just a series of correct choices, and it entails system maintenance, thorough quality control and continuous evaluations in order to ensure stability of the optimal environment.

\section{The Importance of Temperature and Humidity}

It has been reported that embryo metabolism, as well as meiotic function and gamete behavior, are affected by the temperature range the embryo is exposed to $(64,65)$. Commonly, the temperature for embryo culture is regulated at $37^{\circ} \mathrm{C}$ inside an incubator, although Leese et al. have proposed that taking for granted that the optimal culture temperature for embryos is the same as normal core body temperature may not be the case. In many species, the temperature of the fallopian tube is slightly lower than that of the rest of the body, yet it is unclear if that is the case for humans. If it is, then $37^{\circ} \mathrm{C}$ may reflect metabolism dysfunction in early embryonic development (65). On the other hand, a study by Hong et al. comparing effects and safety at temperatures between $36^{\circ} \mathrm{C}$ and $37^{\circ} \mathrm{C}$ showed there to be no difference (66). The issue of optimal temperature is vast and very well documented within the literature supporting the current level to be set at $37^{\circ} \mathrm{C}$, it is however crucial to continue the quest for optimal conditions on all levels involved, including the temperature. Studies like the one by Leese et al. highlight the necessity of questioning what is indeed best.

Temperature and humidity work hand in hand to secure stability in culture. Appropriate levels of humidity ensure a controlled environment regarding the risk of evaporation, which could disturb culture osmolality resulting in catastrophic embryo disturbance $(49,67)$. The matter of dry culture, thereby removing the factor of humidity from the equation of optimal culture conditions, has been raised and argued. Albeit it promising and appealing, recent studies such as the one by Fawzy et al. highlight the need for larger, multicenter randomized trials in strengthening the result of this debate as their data show dry culture to be associated with decreased rates of implantation and clinical pregnancy (68).

\section{Latest Trends}

The nature of culture media and the approach of the IVF laboratory regarding culture conditions are always evolving. Improving conditions and equipment, as well as introducing new technologies, is essential for enhancing success rates.

Close-proximity embryos, decreased media volumes, vertical cultures and group cultures are just a few new trends surfacing. The current practice of static cultures is under exhaustive investigation since it opposes the movement and stimulation an embryo undergoes naturally in vivo. For this reason, dynamic culture platforms providing physical stimulation via shaking, vibrating or rotating, gravity, titling, controlled fluid flow and co-cultures are also some of the new options under examination. All these latest entries into the IVF world are complex and require specific operation as well as time required for a learning curve, adding another level of complexity challenging the practice in the IVF laboratory (69). Microfluidics is a recent innovative technology promising to transform the way an IVF laboratory functions, aiming to optimize culture conditions by manipulating small fluid volumes of media to our advantage. In other words, microfluidic technology tailors the cellular microenvironment as pointed out by Young and Beebe (70). Microfluidic technology promises to expand our perspective regarding gamete and embryo culture as it holds revolutionary potential (71). Challenges of accommodating new ideas and technologies seem to be a mandatory process for establishing a better future for IVF practice. Cell growth and development in advanced stages is reported by Vining and Mooney to be under the immense influence of extrinsic and intrinsic mechanical forces. The fluid surrounding the 
cells throughout early stages and the mechanical forces applied on them appear to have the potential to interfere with embryonic developmental milestones (72). The way these forces interact with genetic and epigenetic events is still unclear. In light of this recent valuable contribution by Vining and Mooney, and the grey zone regarding the effects of mechanical forces, perhaps new trends should be reevaluated. Strict criteria should be in place regarding introduction of relevant technologies to the clinical IVF laboratory as is well presented by Harper et al. (10). The subject of controlling and examining new promising ideas prior to clinically applying them is resurfacing and should be well taken into account and investigated by larger multicenter studies.

The quest of selecting the best embryo has resulted in implementation of ground breaking novel ideas. The oxygen consumption rate was recently proposed for evaluating the embryo's wellbeing. The existing methods from the 1990s for measuring oxygen consumption failed to become a gold standard due to their lack of high sensitivity coupled by their invasive nature. Another innovative method, scanning electrochemical microscopy, employed to measure the oxygen consumption rate failed to excite embryologists as reported by Kurosawa et al. due to the fact that it requires micromanipulation and further training (73). Finally, a new device developed by Kurosawa et al. that included a chip sensing embryo respiration monitoring system achieved an automated measurement of oxygen consumption rate in a satisfactory fashion (73). No matter how exciting these latest trends sound, usability within the clinical spectrum of the IVF laboratory is a required condition that should be seriously accounted for.

The advanced approach of time-lapse technology has entered the IVF world accompanied by plenty of questioning and caution. Time-lapse technology means that sequential images of embryos are recorded during culture with the use of a digital inverted microscope in order to accurately assess the embryo's development (74). Despite the promising outlook, there are numerous studies reporting that time-lapse technology should not be employed, at least not yet, as part of an IVF laboratory's routine due to the lack of adequate data and evidence supporting its efficiency and its contribution towards improving success rates of implantation rates and clinical pregnancies (75-77). Nonetheless, employment of time-lapse technology as a novel approach in the laboratory routine promises to revolutionize embryo culture as we know it, enabling continuous access to the embryo's preimplantation developmental journey and eliminating the factor of exposure while recording precious information. These new technologies not only provide the means to maintain intact culture conditions, in some cases, they also require utilizing different approaches to culturing embryos. For instance, employment of conical dishes is required during culture employing a commercially available time-lapse system called EmbryoScope ${ }^{\mathrm{TM}}$, which may create a unique beneficial surrounding for the embryo in a lowdensity single-embryo culture. Hitherto, validation of the superiority of these technologies regarding culture conditions is pending (78).

\section{Epigenetics}

Essential factors dictating the efficiency of media, such as oxygen, temperature or osmolality, may influence the phenotype of human embryos conceived in an IVF laboratory, through parameters such as morphology, developmental kinetics, physiology and metabolism (79).

The days following fertilization are of great significance. Disruption of a normal event flow could exert a negative effect on the subsequent development of the embryo, and the offspring phenotype. During the preimplantation stages, developmental events may be fine-tuned by epigenetic modifications (80). These crucial modifications manifest as epigenetic perils as they have the potential to influence genomic expression from fertilization all the way through to implantation (81). Any alteration during this sensitive period could have a huge impact on the generations to come $(25,82-85)$.

The gene-expression pattern develops from early embryo developmental stages and is inhibited or activated by internal and external factors. One of the main external factors considered to being capable of altering embryo development and affecting offspring health is the oxygen tension during culture (21). There are various animal studies suggesting that altered gene expression may take place and is majorly dependent on the IVF environment and culture media (8690). Applying these data to humans is challenging and requires careful extrapolation. On this note, Katari and colleagues reported the impact that embryo culture conditions may exert on DNA methylation and gene expression of various tissues such as placenta and cord blood in cases of ART-conceived children (91). Epigenetic differences between the differentiated 'inner' and 'outer' blastomeres, as their polarity is defined, may be attributed to in vitro culture as this study suggests. However, this study further highlights the ambiguity in determining whether these differences in methylation-underlying the epigenetic changes-are a result of ART practice or simply attributed to population characteristics (91).

It has been reported that the percentage of ART-conceived children in developed countries is $1-3 \%$ (92). In light of this considerable percentage, it is imperative to examine the aspects associated with how conditions of ART application may leave an epigenetic print. The fate of the embryo is considered to be affected by modifications during epigenetic reprogramming (93) and is substantially vulnerable to any 
alterations of environmental factors (94). If the beginning of so many new lives takes place in an artificial unprotected or uncontrolled environment of "epigenetic attacks", then what can be anticipated? It has been suggested by Ventura et al. that in the future a possible analysis of the epigenetic profile of IVF born children and adults could reveal and link epigenetic alterations in early stages to pathologies in adult life (93). The follow-up of children is of immense significance. Pediatric data are a must in order to enrich our knowledge and understanding of the underlying mechanisms involved, and to promote an ideal code of practice within the IVF set-up where culture media conditions take the lead role.

\section{Conclusion}

Our goal to minimize environmentally-induced stress inside the IVF laboratory remains in order to provide an optimized culture system for embryo development and to reach most favorable outcomes of ART. Approaches to embryo culture may vary from simplistic in nature and minimally invasive, tried and tested in time, to complicated in application, including novel techniques, equipment and modern concepts presenting promising potential but at times lacking the safety associated with traditional practices. Are we desperately trying to gain control over every single factor in the IVF laboratory for safer results or just simply aiming to be better than nature? (19). Could it be that the embryo's journey in vivo entails more of a rollercoaster regarding the conditions it is subjected to and that our immense effort for stability does not correspond to the reality of the needs of the preimplantation embryo? Data published back in the 1980s proved this point exactly. It was reported that using tap water without any protein supplement was enough to develop embryos to the blastocyst stage $(95,96)$. We have certainly come a long way since then, but the question still stands on whether we may be overthinking and over-emphasizing the details. On the other hand, the very same nature of the ART field and embryology entails the struggle to improve culture conditions and enhance performance and results. Securing a gold standard is key and so is balance between embracing novel approaches such as growth factor addition in media and novel culture platforms and equipment and examining their true potential, efficiency and beneficial nature prior to introducing them into clinical practice. Choosing the most efficient and less interventional approach is a state of mind one should adopt in this era of contradictions, constant innovative new suggestions and struggle to produce better performance statistics for IVF laboratories.

\section{Conflicts of Interest}

The Authors declare that they have no competing interests in regard to this article.

\section{References}

1 Bungum M, Humaidan P and Bungum L: Recombinant human albumin as protein source in culture media used for IVF: A prospective randomized study. Reprod Biomed Online 4(3): 233236, 2002.

2 Cooke S, Quinn P, Kime L, Ayres C, Tyler JP and Driscoll GL: Improvement in early human embryo development using new formulation sequential stage-specific culture media. Fertil Steril 78(6): 1254-1260, 2002.

3 Friedler S, Schachter M, Strassburger D, Esther K, Ron El R and Raziel A: A randomized clinical trial comparing recombinant hyaluronan/recombinant albumin versus human tubal fluid for cleavage stage embryo transfer in patients with multiple IVFembryo transfer failure. Hum Reprod 22(9): 2444-2448, 2007.

4 Dumoulin JC, Land JA, Van Montfoort AP, Nelissen EC, Coonen E, Derhaag JG, Schreurs IL, Dunselman GA, Kester AD and Geraedts JP: Effect of in vitro culture of human embryos on birthweight of newborns. Hum Reprod 25(3): 605-612, 2010

5 Nelissen EC, Van Montfoort AP, Coonen E, Derhaag JG, Geraedts JP, Smits LJ, Land JA, Evers JL and Dumoulin JC: Further evidence that culture media affect perinatal outcome: Findings after transfer of fresh and cryopreserved embryos. Hum Reprod 27(7): 1966-1976, 2012.

6 Nelissen EC, Van Montfoort AP, Smits LJ, Menheere PP, Evers JL, Coonen E, Derhaag JG, Peeters LL, Coumans AB and Dumoulin JC: Ivf culture medium affects human intrauterine growth as early as the second trimester of pregnancy. Human Reprod 28(8): 2067-2074, 2013.

7 El Hajj $\mathrm{N}$ and Haaf T: Epigenetic disturbances in in vitro cultured gametes and embryos: Implications for human assisted reproduction. Fertil Steril 99(3): 632-641, 2013.

8 Mantikou E, Youssef MA, van Wely M, van der Veen F, AlInany $\mathrm{HG}$, Repping $\mathrm{S}$ and Mastenbroek $\mathrm{S}$ : Embryo culture media and IVF/ICSI success rates: A systematic review. Hum Reprod Update 19(3): 210-220, 2013.

9 Quinn P, Kerin JF and Warnes GM: Improved pregnancy rate in human in vitro fertilization with the use of a medium based on the composition of human tubal fluid. Fertil Steril 44(4): 493498, 1985.

10 Harper J, Cristina Magli M, Lundin K, Barratt CL and Brison D: When and how should new technology be introduced into the IVF laboratory? Hum Reprod 27(2): 303-313, 2011.

11 Gardner DK and Lane M: Culture of viable human blastocysts in defined sequential serum-free media. Hum Reprod 13(suppl 3): 148-159, 1998.

12 Summers MC, McGinnis LK, Lawitts JA, Raffin M and Biggers JD: IVF of mouse ova in a simplex optimized medium supplemented with amino acids. Hum Reprod 15(8): 1791-1801, 2000.

13 Karamalegos C and Bolton V: A prospective comparison ofin house'and commercially prepared earle's balanced salt solution in human in vitro fertilization. Hum Reprod 14(7): 1842-1846, 1999.

14 Quinn P: The development and impact of culture media for assisted reproductive technologies. Fertil Steril 81(1): 27-29, 2004.

15 Yoon J, Yoon H-J, Juhn K-M, Ko J-K, Yoon S-H, Ko Y and Lim $\mathrm{J}-\mathrm{H}$ : Application of two different synthetic sequential media for the human IVF-ET program: A prospective, randomized, and comparative study. Clin Exp Reprod Med 38(4): 186-192, 2011. 
16 Mauri AL, Petersen CG, Baruffi RL and Franco JG: Clinical assisted reproduction: A prospective, randomized comparison of two commercial media for icsi and embryo culture. J Assist Reprod Genet 18(7): 378-381, 2001.

17 Staessen C, Janssenswillen C, De Clerck E and Van Steirteghem A: Controlled comparison of commercial media for human in vitro fertilization: Ménézo b2 medium versus Medi-cult universal and BM1 medium. Human Reprod 13(9): 2548-2554, 1998.

18 Sunde A, Brison D, Dumoulin J, Harper J, Lundin K, Magli MC, Van den Abbeel E and Veiga A: Time to take human embryo culture seriously. Hum Reprod 31(10): 2174-2182, 2016.

19 Vajta G, Rienzi L, Cobo A and Yovich J: Embryo culture: Can we perform better than nature? Reprod Biomed Online 20(4): 453-469, 2010.

20 Gardner DK and Lane M: Culture and selection of viable blastocysts: A feasible proposition for human IVF? Hum Reprod Update 3(4): 367-382, 1997.

21 Chronopoulou E and Harper JC: Ivf culture media: Past, present and future. Hum Reprod Update 21(1): 39-55, 2014.

22 Byers SL, Payson SJ and Taft RA: Performance of ten inbred mouse strains following assisted reproductive technologies (ARTS). Theriogenology 65(9): 1716-1726, 2006.

23 Ackerman S, Taylor S, Swanson R and Laurell L: Mouse embryo culture for screening in human IVF. Arch Androl 12: 129-136, 1984.

24 Fleetham JA, Pattinson HA and Mortimer D: The mouse embryo culture system: Improving the sensitivity for use as a quality control assay for human in vitro fertilization. Fertil Steril 59(1): 192-196, 1993.

25 Fleming TP, Kwong WY, Porter R, Ursell E, Fesenko I, Wilkins A, Miller DJ, Watkins AJ and Eckert JJ: The embryo and its future. Biol Reprod 71(4): 1046-1054, 2004.

26 Dumoulin JC, Menheere PP, Evers JL, Kleukers AP, Pieters MH, Bras M and Geraedts JP: The effects of endotoxins on gametes and preimplantation embryos cultured in vitro. Hum Reprod 6(5): 730-734, 1991.

27 Morbeck DE, Krisher RL, Herrick JR, Baumann NA, Matern D and Moyer T: Composition of commercial media used for human embryo culture. Fertil Steril 102(3): 759-766.e759, 2014.

28 Biggers JD: Ethical issues and the commercialization of embryo culture media. Reprod Biomed Online 1(3): 74-76, 2000.

29 Carrasco B, Boada M, Rodríguez I, Coroleu B, Barri PN and Veiga A: Does culture medium influence offspring birth weight? Fertil Steril 100(5): 1283-1288, 2013.

30 Zhu J, Li M, Chen L, Liu P and Qiao J: The protein source in embryo culture media influences birthweight: A comparative study between g1 v5 and g1-plus v5. Hum Reprod 29(7): 13871392, 2014.

31 Khosla S, Dean W, Reik W and Feil R: Culture of preimplantation embryos and its long-term effects on gene expression and phenotype. Hum Reprod Update 7(4): 419-427, 2001.

32 Rooke J, McEvoy T, Ashworth C, Robinson J, Wilmut I, Young $\mathrm{L}$ and Sinclair K: Ovine fetal development is more sensitive to perturbation by the presence of serum in embryo culture before rather than after compaction. Theriogenology 67(3): 639-647, 2007.

33 Eaton J, Lieberman E, Stearns C, Chinchilla M and Racowsky $\mathrm{C}$ : Embryo culture media and neonatal birthweight following IVF. Hum Reprod 27(2): 375-379, 2011.
34 Vergouw CG, Hanna Kostelijk E, Doejaaren E, Hompes PG, Lambalk CB and Schats R: The influence of the type of embryo culture medium on neonatal birthweight after single embryo transfer in IVF. Hum Reprod 27(9): 2619-2626, 2012.

35 Lin S, Li M, Lian Y, Chen L and Liu P: No effect of embryo culture media on birthweight and length of newborns. Hum Reprod 28(7): 1762-1767, 2013.

36 Salter A, Tarling E and Langley-Evans S: Influence of maternal nutrition on the metabolic syndrome and cardiovascular risk in the offspring. Clin Lipidology 4(2): 145-158, 2009.

37 Amosu A and Degun A: Impact of maternal nutrition on birth weight of babies. Biomed Res 25(1): 75-78, 2014.

38 Glujovsky D, Farquhar C, Quinteiro Retamar AM, Alvarez Sedo $\mathrm{CR}$ and Blake D: Cleavage stage versus blastocyst stage embryo transfer in assisted reproductive technology. Cochrane 6: CD002118, 2016.

39 Richter KS: The importance of growth factors for preimplantation embryo development and in vitro culture. Curr Opin Obstet Gynecol 20(3): 292-304, 2008.

40 Kimber S, Sneddon S, Bloor D, El-Bareg A, Hawkhead J, Metcalfe A, Houghton F, Leese H, Rutherford A and Lieberman B: Expression of genes involved in early cell fate decisions in human embryos and their regulation by growth factors. Reproduction 135(5): 635-647, 2008.

41 Brison DR and Schultz RM: Apoptosis during mouse blastocyst formation: Evidence for a role for survival factors including transforming growth factor $\alpha$. Biol Reprod 56(5): 1088-1096, 1997.

42 Fancsovits P, Lehner A, Murber A, Kaszas Z, Rigo J and Urbancsek J: Effect of hyaluronan-enriched embryo transfer medium on IVF outcome: A prospective randomized clinical trial. Arch Gynecol Obstet 291(5): 1173-1179, 2015.

43 Salamonsen LA, Shuster S and Stern R: Distribution of hyaluronan in human endometrium across the menstrual cycle. Cell Tissue Res 306(2): 335-340, 2001.

44 Knudson CB and Knudson W: Hyaluronan-binding proteins in development, tissue homeostasis, and disease. FASEB J 7(13): 1233-1241, 1993.

45 Campbell S, Swann H, Aplin J, Seif M, Kimber S and Elstein M: Fertilization and early embryology: Cd44 is expressed throughout pre-implantation human embryo development. Hum Reprod 10(2): 425-430, 1995.

46 Swain JE: Decisions for the IVF laboratory: Comparative analysis of embryo culture incubators. Reprod Biomed Online 28(5): 535-547, 2014.

47 Fujiwara M, Takahashi K, Izuno M, Duan YR, Kazono M, Kimura $\mathrm{F}$ and Noda Y: Effect of micro-environment maintenance on embryo culture after in vitro fertilization: Comparison of topload mini incubator and conventional front-load incubator. $\mathbf{J}$ Assist Reprod Genet 24(1): 5-9, 2007.

48 Lee M, Grazi R and Seifer D: Incorporation of the cook k-minc incubator and media system into the IVF lab: The future of IVF. J Clin Embryol 13: 21-32, 2010.

49 Swain J: Is there an optimal $\mathrm{pH}$ for culture media used in clinical IVF? Hum Reprod Update 18(3): 333-339, 2012.

50 Goto Y, Noda Y, Mori T and Nakano M: Increased generation of reactive oxygen species in embryos cultured in vitro. Free Rad Biol Med 15(1): 69-75, 1993.

51 Takahashi M: Oxidative stress and redox regulation on in vitro development of mammalian embryos. Jf Reprodd Dev 58(1): 19, 2012 . 
52 Van Blerkom J: Mitochondrial function in the human oocyte and embryo and their role in developmental competence. Mitochondrion 11(5): 797-813, 2011.

53 Calzi F, Papaleo E, Rabellotti E, Ottolina J, Vailati S, Viganò P and Candiani M: Exposure of embryos to oxygen at low concentration in a cleavage stage transfer program: Reproductive outcomes in a time-series analysis. Clin Lab 58(9-10): 997-1003, 2012

54 Fawzy M, Emad M, AbdelRahman MY, Abdelghafar H, Hafez FFA and Bedaiwy MA: Impact of 3.5\% O2 culture on embryo development and clinical outcomes: A comparative study. Fertil Steril 108(4): 635-641, 2017.

$55 \mathrm{Ma}$ Y-Y, Chen H-W and Tzeng C-R: Low oxygen tension increases mitochondrial membrane potential and enhances expression of antioxidant genes and implantation protein of mouse blastocyst cultured in vitro. J Ovarian Res 10(1): 47, 2017.

56 Ghosh J, Coutifaris C, Sapienza C and Mainigi M: Global DNA methylation levels are altered by modifiable clinical manipulations in assisted reproductive technologies. Clin Epigenetics 9(1): 14, 2017.

57 Cohen J, Gilligan A, Esposito W, Schimmel T and Dale B: Ambient air and its potential effects on conception in vitro. Hum Reprod 12(8): 1742-1749, 1997.

58 Hall J, Gilligan A, Schimmel T, Cecchi M and Cohen J: The origin, effects and control of air pollution in laboratories used for human embryo culture. Hum Reprod 13(suppl 4): 146-155, 1998.

59 Khoudja RY, Xu Y, Li T and Zhou C: Better IVF outcomes following improvements in laboratory air quality. J Assist Reprod Genet 30(1): 69-76, 2013

60 Merton J, Vermeulen Z, Otter T, Mullaart E, De Ruigh L and Hasler J: Carbon-activated gas filtration during in vitro culture increased pregnancy rate following transfer of in vitro-produced bovine embryos. Theriogenology 67(7): 1233-1238, 2007.

61 Boone WR, Johnson JE, Locke A-J, Crane MM and Price TM: Control of air quality in an assisted reproductive technology laboratory. Fertil Steril 71(1): 150-154, 1999.

62 Sharmin R and Ray MB: Application of ultraviolet light-emitting diode photocatalysis to remove volatile organic compounds from indoor air. J Air Waste Manage Assoc 62(9): 1032-1039, 2012.

63 Heitmann RJ, Hill MJ, James AN, Schimmel T, Segars JH, Csokmay JM, Cohen J and Payson MD: Live births achieved via IVF are increased by improvements in air quality and laboratory environment. Reprod Biomed Online 31(3): 364-371, 2015.

64 Sun X-F, Wang W-H and Keefe DL: Overheating is detrimental to meiotic spindles within in vitro matured human oocytes. Zygote 12(1): 65-70, 2004.

65 Leese HJ, Baumann CG, Brison DR, McEvoy TG and Sturmey RG: Metabolism of the viable mammalian embryo: Quietness revisited. Mol Hum Reprod 14(12): 667-672, 2008.

66 Hong KH, Lee H, Forman EJ, Upham KM and Scott RT: Examining the temperature of embryo culture in in vitro fertilization: A randomized controlled trial comparing traditional core temperature $\left(37^{\circ} \mathrm{C}\right)$ to a more physiologic, cooler temperature $\left(36^{\circ} \mathrm{C}\right)$. Fertil Steril 102(3): 767-773, 2014.

67 Lane M, Mitchell M, Cashman KS, Feil D, Wakefield S and Zander-Fox DL: To QC or not to QC: The key to a consistent laboratory? Reprod Fertil Dev 20(1): 23-32, 2007.

68 Fawzy M, AbdelRahman MY, Zidan MH, Hafez FFA, Abdelghafar $\mathrm{H}$, Al-Inany $\mathrm{H}$ and Bedaiwy MA: Humid versus dry incubator: A prospective, randomized, controlled trial. Fertil Steril 108(2): 277-283, 2017.

69 Swain J and Smith G: Advances in embryo culture platforms: Novel approaches to improve preimplantation embryo development through modifications of the microenvironment. Hum Reprod Update 17(4): 541-557, 2011.

70 Young EW and Beebe DJ: Fundamentals of microfluidic cell culture in controlled microenvironments. Chem Soc Rev 39(3): 1036-1048, 2010.

71 Smith G, Swain J and Bormann C: Microfluidics for gametes, embryos, and embryonic stem cells. In: Seminars in Reproductive Medicine. Thieme Medical Publishers pp. 5-14, 2011.

72 Vining KH and Mooney DJ: Mechanical forces direct stem cell behaviour in development and regeneration. Nat Rev Mol Cell Biol 18(12): 728-742, 2017.

73 Kurosawa H, Utsunomiya H, Shiga N, Takahashi A, Ihara M, Ishibashi M, Nishimoto M, Watanabe Z, Abe $\mathrm{H}$ and Kumagai J: Development of a new clinically applicable device for embryo evaluation which measures embryo oxygen consumption. Hum Reprod 31(10): 2321-2330, 2016.

74 Racowsky C, Kovacs P and Martins WP: A critical appraisal of time-lapse imaging for embryo selection: Where are we and where do we need to go? J Assist Reprod Genet 32(7): 1025$1030,2015$.

75 Armstrong S, Vail A, Mastenbroek S, Jordan V and Farquhar C: Time-lapse in the IVF-lab: How should we assess potential benefit? Hum Reprod 30(1): 3-8, 2014.

76 Bhide P, Maheshwari A, Cutting R, Seenan S, Patel A, Khan K and Homburg R: Time lapse imaging: Is it time to incorporate this technology into routine clinical practice? Hum Fertil 20(2): 74-79, 2017.

77 Chen M, Wei S, Hu J, Yuan J and Liu F: Does time-lapse imaging have favorable results for embryo incubation and selection compared with conventional methods in clinical in vitro fertilization? A meta-analysis and systematic review of randomized controlled trials. PloS One 12(6): e0178720, 2017.

78 Kieslinger DC, De Gheselle S, Lambalk CB, De Sutter P, Kostelijk EH, Twisk JW, Van Rijswijk J, Van den Abbeel E and Vergouw CG: Embryo selection using time-lapse analysis (early embryo viability assessment) in conjunction with standard morphology: A prospective two-center pilot study. Hum Reprod 31(11): 2450-2457, 2016.

79 Gardner D and Kelley R: Impact of the IVF laboratory environment on human preimplantation embryo phenotype. J Dev Orig Health Dis 8(4): 418-435, 2017.

80 Smith ZD, Chan MM, Mikkelsen TS, Gu H, Gnirke A, Regev A and Meissner A: A unique regulatory phase of DNA methylation in the early mammalian embryo. Nature 484(7394): 339-344, 2012.

81 Brower V: Epigenetics: Unravelling the cancer code. Nature 471(7339): S12-S13, 2011.

82 Denomme MM and Mann MR: Genomic imprints as a model for the analysis of epigenetic stability during arts. Reproduction 144(4): 393-409, 2012.

83 Van Montfoort A, Hanssen L, De Sutter P, Viville S, Geraedts J and De Boer P: Assisted reproduction treatment and epigenetic inheritance. Hum Reprod Update 18(2): 171-197, 2012.

84 Feuer S, Camarano L and Rinaudo P: Art and health: Clinical outcomes and insights on molecular mechanisms from rodent studies. Mol Hum Reprod 19(4): 189-204, 2012. 
85 Thompson JG, Kind KL, Roberts CT, Robertson SA and Robinson JS: Epigenetic risks related to assisted reproductive technologies: Short-and long-term consequences for the health of children conceived through assisted reproduction technology: More reason for caution? Hum Reprod 17(11): 2783-2786, 2002.

86 Morgan HD, Jin XL, Li A, Whitelaw E and O'Neill C: The culture of zygotes to the blastocyst stage changes the postnatal expression of an epigentically labile allele, agouti viable yellow, in mice. Biol Reprod 79(4): 618-623, 2008.

87 Rinaudo P and Schultz RM: Effects of embryo culture on global pattern of gene expression in preimplantation mouse embryos. Reproduction 128(3): 301-311, 2004.

88 Fernández-Gonzalez R, Moreira P, Bilbao A, Jiménez A, PérezCrespo M, Ramírez MA, De Fonseca FR, Pintado B and Gutiérrez-Adán A: Long-term effect of in vitro culture of mouse embryos with serum on mrna expression of imprinting genes, development, and behavior. Proc Natl Acad Sci USA 101(16): 5880-5885, 2004.

89 Mahsoudi B, Li A and O'Neill C: Assessment of the long-term and transgenerational consequences of perturbing preimplantation embryo development in mice. Biol Reprod 77(5): 889-896, 2007.

90 Calle A, Miranda A, Fernandez-Gonzalez R, Pericuesta E, Laguna $\mathrm{R}$ and Gutierrez-Adan A: Male mice produced by in vitro culture have reduced fertility and transmit organomegaly and glucose intolerance to their male offspring. Biol Reprod 87(2): 31-39, 2012
91 Katari S, Turan N, Bibikova M, Erinle O, Chalian R, Foster M, Gaughan JP, Coutifaris C and Sapienza C: DNA methylation and gene expression differences in children conceived in vitro or in vivo. Human molecular genetics 18(20): 3769-3778, 2009.

92 Manipalviratn S, DeCherney A and Segars J: Imprinting disorders and assisted reproductive technology. Fertil Steril 91(2): 305-315, 2009.

93 Ventura-Juncá P, Irarrázaval I, Rolle AJ, Gutiérrez JI, Moreno RD and Santos MJ: In vitro fertilization (IVF) in mammals: Epigenetic and developmental alterations. Scientific and bioethical implications for IVF in humans. Biol Res 48(1): 68, 2015.

94 Faulk C and Dolinoy DC: Timing is everything: The when and how of environmentally induced changes in the epigenome of animals. Epigenetics 6(7): 791-797, 2011.

95 George MA, Braude PR, Johnson MH and Sweetnam DG: Quality control in the IVF laboratory: In-vitro and in-vivo development of mouse embryos is unaffected by the quality of water used in culture media. Hum Reprod 4(7): 826-831, 1989.

96 Silverman IH, Cook CL, Sanfilippo JS, Yussman MA, Schultz GS and Hilton FH: Ham's F-10 constituted with tap water supports mouse conceptus development in vitro. J Assist Reprod Genet 4(3): 185-187, 1987.

Received February 13, 2018

Revised March 13, 2018

Accepted March 20, 2018 